Article available at http://www.parasite-journal.org or http://dx.dol.org/10.1051/parasite/2008153310

\title{
Plant-NemATOdE INTERACTIONS: ENVIRONMENTAL SIGNALS DETECTED BY THE NEMATODE'S CHEMOSENSORY ORGANS CONTROL CHANGES IN THE SURFACE CUTICLE AND BEHAVIOUR
}

\author{
CURTIS R.H.C.*
}

\section{Summary:}

Plant parasitic nematodes have developed the capacity to sense and respond to chemical signals of host origin and the ability to orientate towards plant roots enhances the nematode's chance of survival. Root exudates contain a range of compounds which mediate belowground interactions with pathogenic and beneficial soil organisms. Chemical components of root exudates may deter one organism while attracting another and these compounds alter nematode behaviour and can either attract nematodes to the roots or result in repellence, motility inhibition or even death. In vitro, plant signals present in root exudates, trigger a rapid alteration of the surface cuticle of Meloidogyne incognita and the same changes were also induced by indole-acetic acid (IAA). IAA binds to the chemosensory organs of $M$. incognita and it is possible that IAA acts as a signal that orientates the nematode on the root surface in the rhizosphere and/or inside the root tissue and thereby promotes nematode infection.

KEY WORDS : Meloidogyne spp., indole-acetic acid, plant signals, cuticle, amphids.

\section{PLANT-NEMATODE INTERACTIONS IN THE RHIZOSPHERE}

$\mathrm{P}$ lant parasitic nematodes are of considerable economic importance world-wide, Meloidogyne spp. (root-knot nematodes) and Globodera spp. (potato cyst nematodes) being particularly destructive, contributing greatly to an estimated annual yield loss of $\$ 100$ billion.

During co-evolution with the host plant, parasitic nematodes have developed the capacity to recognise and respond to chemical signals of host origin. Understanding the complexity of the molecular signal exchange and response during the early stages of the host-parasite interactions is important to identify vulnerable points in the parasite life cycle that can be targeted to disrupt nematode-host recognition. Nematode invasion of roots and the migration to the nematode feeding sites result in changed root architecture and significant reductions in nutrient and water uptake and consequent crop yields.

* Nematode Interactions Unit, Rothamsted Research, Harpenden, AL5 2JQ, UK.

Tel.: +44 (0)1 5827633133 - Fax: +44 (0)1 582760981 .

E-mail: rosane.curtis@bbsrc.ac.uk
The plant-nematode relationship is governed by a complex network of interactions resulting in the formation of feeding sites. The infective second-stage juveniles (J2) of Globodera spp. penetrate roots and migrate intracellularly towards the vascular cylinder and establish an intimate nutritional relationship with their host through the development of syncytial feeding sites. In contrast, J2 of Meloidogyne spp. after penetrating the root epidermis migrate intercellularly between cortical cells until they find a suitable root cell to form their feeding site. Root cells around the nematode's head are stimulated to go through repeated rounds of mitosis uncoupled from cytokinesis, leading to multinucleated giant cells (Gheysen \& Fenoll, 2002). Ultimately, the formation of the feeding site is the result of nematode-induced alterations in plant gene expression (reviewed in Gheysen \& Fenoll, 2002). Feeding cell formation is presumably initiated in response to signal molecules released by the $\mathrm{J} 2$, but the nature of the primary stimulus is unknown. The most widely held hypothesis is that the necessary metabolic reprogramming of root cells is triggered by specific nematode secretions, which presumably interact with membrane or cytoplasmic receptors in the plant to switch on cascades of gene expression that alter cell development (Williamson \& Hussey, 1996). Syncytia and giant cells require repeated stimulation from the nematode to maintain their function and cyst and root-knot nematodes depend entirely on functional feeding cells to complete their life cycles. Endoparasitic plant nematodes do not feed during their migrations in soil and in roots and their survival depends on finding a food source or sexual partner without unnecessary energy expenditure. They rely on stored lipid reserves to provide the energy for their movement. As with heminths, these infective stages recognise their hosts via complex sequences of behavioural patterns in response to various environmental cues (Haas et al., 1997; Haas, 2003). Plant signals are essential for nematodes to locate hosts and feeding sites, nematodes with $>60 \%$ of their lipid reserves depleted are no longer capable of directed movement (Robinson et al., 1987). However, the identity of the plant signals involved in key stages of the plant-nematode interactions are not yet clearly defined. 
Many parasitic nematodes enter their hosts by active invasion and their transmission success is often based on a mass production of infective stages which show a highly specific host-finding behaviour. These infective stages recognise their hosts via complex sequences of behavioural patterns with which they successfully respond to various environmental and host cues (Haas et al, 1997; Haas, 2003). After invasion animal parasites navigate through their host's tissues to their very specific microhabitats and some of these migrating parasitic stages can follow chemical gradients of skin and blood compounds (Haas et al., 2002). It is suggested that the high complexity, specificity and diversity of hostrecognition strategies devised by parasites are important determinants in the evolution of their life cycles.

\section{BELOWGROUND CHEMICAL COMMUNICATION: ROOT EXUDATES AND HOST RECOGNITION}

T he rhizosphere encompasses the soil within millimetres of a plant root where complex biological and ecological processes occur. Plant roots exude a range of compounds into the rhizosphere which mediate belowground interactions with pathogenic and beneficial soil organisms. Root exudates include secretion of ions, free oxygen and water, enzymes, mucilage and a diverse array of primary and secondary metabolites (Bertin et al., 2003). Although, the functions of most root exudates have not been determined, several compounds play important roles in biological processes (reviewed in Bais et al., 2006). Root compounds are secreted into the surrounding rhizosphere or released from root border cells which separate from the roots as they grow.

Chemical components of root exudates may deter one organism while attracting another and these compounds alter nematode behaviour (Fig. 1) and can either attract nematode to the roots or result in repellence, motility inhibition or even death (Robinson, 2002; Wuyts et al., 2006; Zhao et al., 2000). It seems that a combination of signals in a given set of exudates determines the nematode behaviour in a given plant-nematode interaction. Following fractionation of cucumber root exudates, fractions with both repellent and attractant activity to Meloidogyne incognita were isolated (Castro et al., 1988). Root border cells play an important role in protecting the root tip from infection by acting as a natural trap for pathogenic organisms (Wuyts et al., 2006; Zhao et al., 2000). Indeed, root cap exudates, whose secretions include enzymes, antibiotics, other soluble molecules and high molecular weight mucilage that encases border cells. They repelled both plant nematodes and the free-living nematode Caenorhabidtis elegans and producing a reversible state of immobility

\section{Belowground chemical communication Root exudates and host recognition}

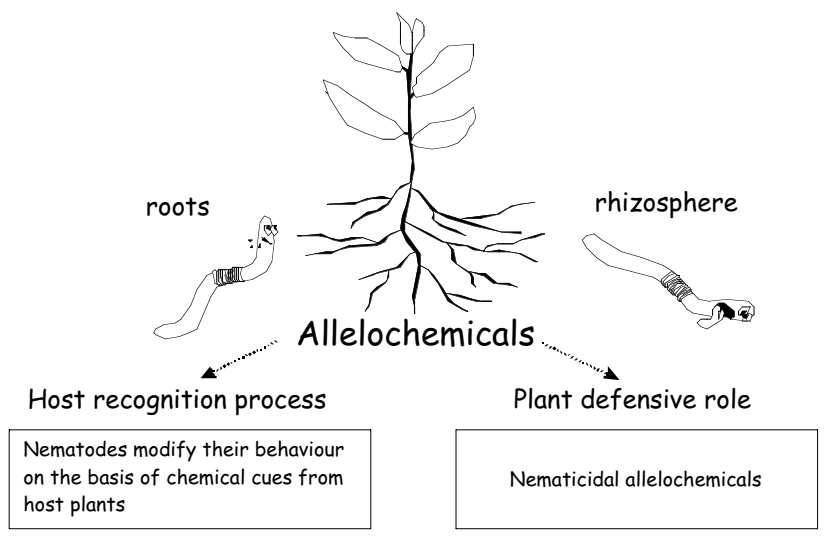

Fig. 1. - Allelochemicals can be found in parts of the plant and can also be secreted as root exudates or released as volatile compounds. They mediate chemical interactions between two plants as well as plant-pathogen chemical communication. Attraction, penetration and feeding behaviour of plant parasitic nematodes involve molecular communication between the nematode and respective plants. Root exudates can induce attraction, repellence, inhibition and hatching stimulation.

in these nematodes (Hubbard, et al., 2005; Wuyts et al., 2006; Zhao et al., 2000). Therefore, localised sites on the root surface and rhizosphere drive population dynamics. This ability of the root tip to deliver products that temporally immobilises nematodes could play a role in plant defence for abiotic and biotic factors. Indeed, the root cap provides physical protection for the apical meristem especially against the soil pathogens (Zhao et al., 2000). This phenomenon might account in part, for the fact that the normal site of infection by nematodes is behind the root tip, just past the region where border cells are released (Zhao et al., 2000).

The role of plant signals in synchronising host and parasite life-cycle is best illustrated with the two species of potato cyst nematodes (Globodera spp.), as these nematodes almost completely depend on root exudates for hatching. Various hatching factors have been identified in crop plants and these compounds are potential candidates for agrochemical use (Devine \& Jones, 2001). Hatching is an important step in the nematode life-cycle leading to parasitism and this dependence of the Globodera spp. on hatching factors have been exploited to reduce the number of nematodes in infested fields (Timmermans et al., 2007). Solanum sisymbriifolium is used successfully as a trap crop for potato cyst nematodes in Europe, as this plant stimulates hatch of second stage juveniles but does not support their development and is completely resistant to G. pallida and G. rostochiensis (Timmermans et al., 2007).

The ability to orientate towards plant roots enhances the nematode survival. Long distance attractants' can enable nematodes to locate roots; whilst attractants that cause 
the nematode to move to individual host roots may be termed as "short distance attractants" and the orientation to the preferred site of invasion in the root tip might be mediated by "local attractants" (reviewed in Perry, 2001). So far, only $\mathrm{CO}_{2}$ has been identified as a prime long distance attractant for plant parasitic nematodes, including M. incognita (Robinson, 2002). Additional attractants are aminoacids, sugars and metabolites (Bird, 1959; Robinson, 2002; Perry, 2001; Prot, 1980). It has been suggested that nematodes orientate to an electrical potential gradient at the elongation zone of the root tip (Bird, 1959).

The chemotactic response of Bursaphelenchus xylophilus, the pine wood nematode, to a variety of chemicals varied according to the nematodes lipid content. Nematodes with the lowest lipid content were attracted to the pine wood volatile $\alpha$-myrcene, while nematodes with the highest lipid content were attracted to a toluene hydrocarbon in the cuticle of the beetle vector for the nematode (Stamps \& Linit, 2001). Therefore, nematode lipid contents seem to be an important factor in the nematode's response to host signals. Plant roots also trigger $C$. elegans behavioural response by emitting volatile signals attracting nematodes to the root proximity (Horiuchi et al., 2005).

Plant roots also produce allelochemicals to defend the plant against potential soil-borne pathogens and a number of plant secondary metabolites have been shown to function as nematode antagonists (reviewed in Guerena, 2006) For example, the triterpenoid compound, cucurbitacin A, from cucumber plants repels nematodes and a $\beta$-terthienyl compound from Tagetes erecta is repellent and nematotoxic (Castro et al., 1988). Allelochemicals present in the root exudates of maize such as cyclic hydroxamic acid, have been shown to affect the behaviour of Pratylenchus zea, M. incognita and Xiphinema americanum. The methoxylated hydroxamic acid (DIMBOA, dihydroxy-7-methoxy-1,4-benzoxaxin-3(4H) increased attraction and its degradation product (MBOA, 6-methoxy-2-benzoxazolinone) induced repellence to roots (Friebe et al., 1997; Zasada et al., 2005).

Root exudates not only contain compounds that induce nematode hatching, attraction and repellence to roots but also compounds that induce characteristic nematode exploratory behaviour, including stylet thrusting; release of secretions in preparation for root penetration; aggregation and increase in nematode mobility (Clarke \& Hennessy, 1984; Curtis, 2007; Grundler et al., 1991; Robinson, 2002).

\section{SURFACE CUTICLE CHANGES IN RESPONSE TO ENVIROMENTAL SIGNALS}

he epicuticle forms the outer cuticular layer of nematodes, which is covered in many species by a fuzzy coating material termed the "surface coat" (SC). The SC is composed mainly of proteins, carbohydrates and lipids (Spiegel \& McClure, 1995). The nematode surface coat, being the outermost layer of the cuticle, is considered one of the major targets for developing new control strategies against plant-parasitic nematodes. Nematode secretions and surface coat antigens are likely to be the first signals perceived by the plant and they probably have important roles in the host-parasite relationship (Jones \& Robertson, 1997). They may help with the initial penetration, migration of the second stage juveniles in the plant tissues and digestion of host cell contents (Williamson \& Hussey, 1996) and are also probably involved in the protection of the nematode from plant defense responses (Prior et al., 2001; Robertson et al., 2000). Interfering with nematode movement affect nematode invasion of plants (Sharon et al, 2002; Fioretti et al., 2002) and can render nematodes vulnerable to anti-nematode control strategies in the rhizosphere and/or root tissue.

Nematodes can rapidly change their surface composition in response to environmental signals, which may enable animal parasitic nematodes to escape host immune responses and free-living nematodes to escape pathogenic infections (Olsen et al., 2007; Proudfoot et al., 1993). Surface composition can also change within a single stage during entry of parasitic nematodes into a new host or host tissue (Modha et al., 1995; Proudfoot et al., 1993). One of the most interesting features of the nematode SC is its dynamic nature; there is a continuous turn-over of the surface associated antigens, which are shed and replaced (Blaxter \& Robertson, 1998). In plant-parasitic nematodes this was demonstrated for the pre-parasitic juveniles of Meloidogyne spp. (Lin \& McClure, 1996; Spiegel et al., 1995), the importance of these changes in the surface coat for host-parasite interactions is less well understood.

Considerable similarities were found in the surface cuticle of certain plant and animal parasitic nematodes (Lopez de Mendoza et al., 1999). Monoclonal antibodies (mAb) raised to excreted/secreted (ES) products of the plant parasitic nematode Heterodera avenae was also shown to bind to secretions from the cuticle surface and amphids of Meloidogyne incognita (Curtis, 1996) and to the surface coats of the animal parasites Trichinela spiralis and Haemonchus contortus (Curtis, 1996; Lopez de Mendonza et al., 1999).

\section{ROOT EXUDATES AND PHYTOHORMONES INDUCE CHANGES IN THE SURFACE CUTICLE OF NEMATODES}

$\mathrm{P}$ lant signals present in root exudates, trigger a rapid alteration of the surface cuticle of Meloidogyne incognita and Globodera rostochiensis 
and the same changes were also induced by phytohormones, in particular indole-acetic acid (IAA) to $M$. incognita but not G. rostochiensis (Akhkha et al., 2002, 2004; Curtis, 2007; Lopez de Mendoza et al., 2000). As the former nematode can infect a large range of host plants it was not surprising that responded to such a general plant compound. This surface change induced by plant signals might allow $M$. incognita to adapt and survive plant defence processes. However, more specific host cues from root exudates of solanaceous plants were responsible for increasing the lipophilicity of the surface cuticle of infective juveniles of Globodera species (Akhkha et al., 2002). In vitro, IAA has also been shown to induce the production of nematode secretions (Duncan et al., 1995) and an increase in nematode mobility (Curtis, 2007). Globodera pallida (Duncan et al., 1995) but not Meloidogyne spp. (R. Curtis unpublished data) was shown to have an immunological cross-reactivity to the maize auxin binding protein, indicating that these sedentary plant parasitic nematodes with their very different host specificities may respond differently to IAA.

Molecules present in root exudates, including IAA may act as environmental signals to induce these behavioural changes and therefore play a vital role in the host-recognition processes for sedentary plant parasitic nematodes. Exogenous application of IAA shifted the response of resistant tomato plants towards susceptibility (Dropkin et al., 1969; Sawhney \& Webster, 1975) and these results support the importance of phytohormones for plant-nematode interactions.

The nematode life-cycle may be synchronised by the IAA concentration in the root tissues. The preferred site for root invasion is at the elongation zone of growing tips (Fig. 2a \& b), where higher levels of IAA fluxes have been recorded (Mancuso et al., 2005). Transport of IAA in plants occurs from the shoot to the root tip and then laterally to the epidermis. The $\mathrm{J} 2$ of Meloidogyne spp. migrate inside the roots from the elongation zone to the tip and thence to the stele in the opposite direction to the IAA transport gradient (reviewed in von Mende, 1997). High concentrations of IAA have also been detected around the root cells surrounding the nematode head (Karczmark et al., 2004). It is possible that IAA acts as a signal that orientates the nematode on the root surface in the rhizosphere and inside the root tissue and thereby promotes nematode infection.

However, whether nematodes perceive IAA as host cues in the rhizosphere, at the root surface and/or in the root tissue is not known. Nematodes may encounter an IAA gradient inside roots and free IAA has been detected up to concentrations of $1 \mu \mathrm{M}$ in phloem exudates (Friml, 2003). But, yet it is not known if nematodes are affected by IAA from plant origin in the rhizosphere and so far, only auxin conjugates such as IAA methyl-glutamate have been shown to be present in root exudates of Arabidopsis thatiana (Narasimham et al., 2003).

\section{NEMATODES DETECT ENVIRONMENTAL SIGNALS PRESENT IN THE RHIZOSPHERE USING THEIR CHEMOSENSORY ORGANS}

T The nematode nervous system is the conduit between stimulus, reception and behavioural output and multicellular animals use their chemical senses to trigger complex behaviours and developmental processes. The main nematode chemosensory organs involved in host-recognition processes are two bilaterally symmetrical amphids (Fig. 3) in the nematode head and two paired pore-like phasmids located in the lateral field of the nematode tail. Nematodes have the ability to chemo-orientate using a combination of head to tail chemosensory sensors to simultaneously compare the intensities of the stimulus across their body length (Hilliard et al., 2002).

The amphids contain a number of dendritic processes which are surrounded by secretions produced by the
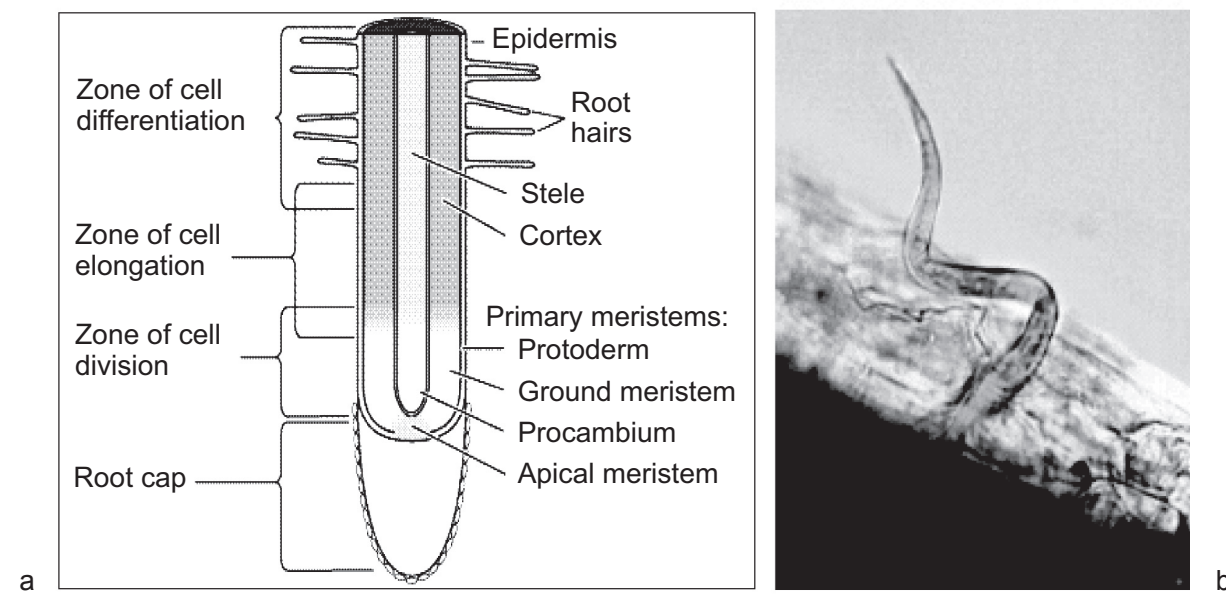

Fig. 2 a \& b. - a. Root anatomy: the zone of elongation is prefered site of penetration for sedentary plant parasitic nematodes and chemical gradients can be used as orientation markers for short distances on the root surface and within the root tissue. b. Photograph showing $M$. incognita second stage juveniles invading a tomato root at the zone of elongation. 


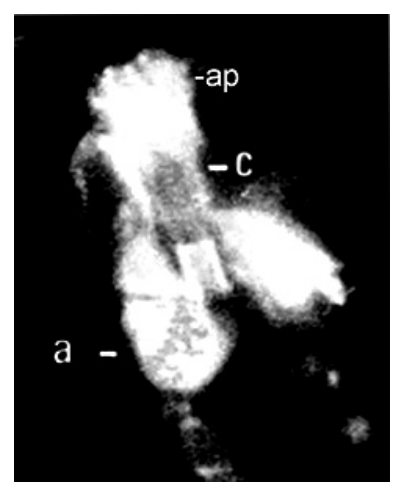

Fig. 3. - Immunofluorescence photograph of a cryostat section of the anterior head of the second stage juvenile of Globodera pallida, the section was probed with a monoclonal antibody reactive with the nematode amphids. $\mathrm{a}=$ amphidial glands; $\mathrm{c}=$ amphidial canal; ap $=$ amphidial pores.

glandular sheath cell. Disruption of chemoresponses render nematodes disorientated in the soil and unable to find a host (Fioretti et al., 2002; Perry 2005; Zuckerman, 1983). The nematicide aldicarb disturbed nematode orientation and caused changes in the ultrastructure of the amphidial sheath cell of affected nematodes (Trett \& Perry, 1985). Also, interference with nematode chemoreception, by blocking amphidial secretion using monoclonal and polyclonal antibodies, stopped nematode movement and significantly reduced infection of plants (Fioretti et al., 2002; Sharon et al., 2002; Stewart et al., 1993).

Chemo-orientation in a concentration gradient is vital for nematode survival and is essential for detection of host plant exudates, food stimulants, food deterrents and sex pheromones (http://www.wormbook.org). The free living nematode $C$. elegans changes its surface in response to environmental chemical signals detected by the nematodes's chemosensory organs (Olsen et al., 2007). Plant nematodes also rely on chemoreception, e.g. to find a host in the soil, and when a root is encountered, its surface is explored for a suitable penetration site. IAA binds to the chemosensory organs of $M$. incognita (Curtis, 2007). IAA has been implicated in the recognition process of host plants by fungi. During this interaction Saccharomyces cerevisiae differentiates into an invasive form, which enables infection. In this case IAA induces morphogenetic changes, such as adhesion and filamentation, which are mediated by fungal transcription factors and a family of transporters, which are upregulated in the fungus. These transporters are members of the amino acid/auxin:proton symport permeases (AAAP) superfamily, which includes Arabidopsis AUX1, a putative IAA transporter from plants, and families related to neurotransmitter transporters such as the $\delta$ aminobutyric acid (GABA) transporters in C. elegans (McIntire et al., 1997; Prusty et al., 2004). Indeed, GABAergic neurons act to control the body and enteric muscles necessary for different behaviours in C. elegans (McIntire et al., 1997). Amino acid and neurotransmitter transporters are highly conserved in animals and plants and they function in vesicle-based cell-to-cell transport (Baluska et al., 2003; Chen et al., 2001).
Plant, animals and pathogens share elements such as fatty acids, proteins, neurotransmitters and in some of the signalling machinery underlying responsiveness to the environment (Schultz, 2002). Therefore, it is not surprising that plants produce signalling molecules for which there are nematode receptors/binding proteins. Nematode signalling components present in the chemosensory organs are in contact with the external environment and therefore are exposed to antagonistic molecules that could block their interaction with host cues. Understanding the signalling and perception processes occurring in plant-nematode interactions will reveal targets for chemical or genetic intervention.

\section{ACKNOWLEDGEMENTS}

R othamsted Research receives grant-aided support from the Biotechnology and Biological Sciences Research Council of the UK.

\section{REFERENCES}

Akhkha A., Kusel J., Kennedy M. \& Curtis R.H.C. Effects of phytohormones on the surfaces of plant parasitic nematodes. Parasitology, 2002, 125, 165-175.

Akhkha A., Curtis R, Kennedy M. \& Kusel J. The potential signaling pathways which regulate surface changes induced by phytohormones in the potato cyst nematode ( $G l o-$ bodera rostochiensis). Parasitology, 2004, 128, 533-539.

Bais H.P., Weir T.L., Perry L.G., Gilroy S. \& Vivanco J.M. The role of root exudates in rhizosphere interactions with plants and other organisms. Annual Review Plant Biology, 2006, 57, 233-266.

Baluska F., Volkman, D. \& Menzel D. Plant synapses: actinbased domains for the cell-to-cell communication. Trends in Plant Science, 2005, 10, 106-111.

Bertin C., Yang X.H. \& Weston L.A. The role of root exudates and allelochemicals in the rhizosphere. Plant Soil, 2003, 256, 67-83.

Blaxter M. \& Robertson W. The cuticle, in: Free-living and plant parasitic nematodes Perry R.N. CAB International, Wallingford, UK, 1999, 25-48.

BIRD F. The attractiveness of roots to the plant parasitic nematodes Meloidogyne javanica and M. hapla. Nematologica, 1959, 42 322-335.

Castro C.E., Belser N.O., McKinney H.E. \& Thomason I.J. Quantitative bioassay for chemotaxis with plant parasitic nematodes. Attractant and repellent fractions for Meloidogyne incognita from cucumber roots. Journal of Chemical Ecology, 1988, 15, 1297-1309.

Chen L., Ortiz-Lopez A., Jung A. \& Bush D.R. ANT1, an aromatic and neutral amino acid transporter in Arabidopsis. Plant Physiology, 2001, 125, 1813-1820.

Clarke A.J. \& Hennessy J. Movement of Globodera rostochiensis juveniles stimulated by potato root exudate. Nematologica, 1984, 30, 206-212. 
CuRTIS R.H.C. Identification and in situ and in vitro characterisation of secreted proteins produced by plant-parasitic nematodes. Parasitology, 1996, 113, 589-593.

CurTis R.H.C. Do phytohormones influence nematode invasion and feeeding site establishment? Nematology, 2007, 9, 155-160.

Devine K.J. \& Jones P.W. Potato cyst nematode hatching activity and hatching factors in inter-specific Solanum hybrids. Nematology, 2001, 3, 141-149.

Dropkin V.H., Helgeson J.P. \& Upper C.D. The hypersensitivity reaction of tomatoes resistant to Meloidogyne incognita: Reversal by cytokinins. Journal of Nematology, 1969, 1, 5561.

Duncan L.H., Robertson W.M., Kusel R.J \& Phillips M.S. A putative nematode auxin binding protein from the potato cyst nematode Globodera pallida. Nematropica, 1996, 26, 259.

Fioretti L., Porter A., Haydock P.J. \& Curtis R.H.C. Monoclonal antibodies reactive with secreted-excreted products from the amphids and the cuticle surface of Globodera pallida affect nematode movement and delay invasion of potato roots. International Journal for Parasitology, 2002, 32, 1709-1718.

Friebe A., Lever W., Sikora R. \& Schnabl H. Allelochemical in root exudates of maize. Effects on root lesion nematode Pratylenchus zea, in: Phytochemical signals and plantmicrobe interactions. Recent Advances in Phytochemistry, 1997, 32, 71-93.

FRIML J. Auxin transport-shaping the plant. Current Opinion in Plant Biology, 2003, 6, 7-12

Gheysen G. \& Fenoll C. Gene expression in nematode feeding sites. Annual Review of Phytopathology, 2002, 40, 191219.

Grundler F., Schnibbe L. \& Wyss U. In vitro studies on the behaviour of second stage juveniles of Heterodera schachtii in response to host plant root exudates. Parasitology, 1991, 103, 149-155.

GuERENA M. Nematodes: alternative control. National sustainable agriculture information service. ATTRA publication \#IP287, 2006, 1-20.

HaAs W. Parasitic worms: strategies of host finding, recognition and invasion. Zoology, 2003, 106, 349-364.

HaAs W., Diekhoff D., Koch K., Schmalfuss G. \& Loy C. Schistosoma mansoni cercariae: stimulation of acetabular gland secretion is adapted to the chemical composition of mammalian skin. Journal of Parasitology, 1997, 83, 1079-1085

Haas W., Grabe K., Geis C., Pach T., Stoll K., Fuchs M., Haberl A \& LOY C. Recognition and invasion of human skin by Schistosoma mansoni cercariae: the key-role of Larginine. Parasitology, 2002, 124, 153-167.

Hilliard M.A., Bargmann C.I. \& Bazzicalupo P. C. elegans responds to chemical repellents by integrating sensory inputs from the head and tail. Current Biology, 2002, 12, 730-734

Horiuchi J., Prithiviraj B., Bais H., Kimball B.A. \& Vivanco J.M. Soil nematodes mediate positive interactions between legume plants and rhizobium bacteria. Planta, 2005, 222 848-857.
Hubbard J., Flores-Lara Y., Schmitt M., McClure M.A., Stock P. \& HAwES M. Increased penetration of host roots by nematodes after recovery from quiescence induced by root cap exudate. Nematology, 2005, 7, 321-331.

Jones J. \& Robertson W. Nematodes secretions, in: Aspects of plant parasitic nematode interactions. Fenoll C., Grundler F.M. \&Ohl S.A. (eds), Kluwer Academic Publishers, The Netherlands, 1997, 98-106.

Karczmark A., Overmars H., Helder J. \& Goverse A. Feeding cell development by cyst and root-knot nematodes involves a similar early, local and transient activation of a specific auxin-inducible promoter element. Molecular Plant Pathology, 2004, 5, 343-346.

LiN H. \& MCCLuRE M.A. Surface coat of Meloidogyne incognita. Journal of Nematology, 1996, 28, 216-224.

Lopez de Mendoza M.E., CurTis R.H.C. \& Gowen S. Identification and characterisation of excreted-secreted products and surface coat antigens of animal and plant parasitic nematodes. Parasitology, 1999, 118, 397-405.

Lopez de Mendoza M.E., Modha J., Roberts C., Curtis R.H.C. \& KusEL J. Observations of the changes of the surface cuticle of parasitic nematodes using fluorescent probes. Parasitology, 2000, 120, 203-209.

Mancuso S., Marras A. M., Magnus V. \& Baluska F. Noninvasive and continuous recordings of auxin fluxes in intact root apex with a carbon nanotube-modified and self-refering microelectrode. Analytical Biochemistry, 2005, 341, 344-351.

Mcintire S., Remer R., Schuske K., Edwards R.H. \& JorgenSEN E.M. Identification and characterisation of the vesicular GABA transporter. Nature, 1997, 389, 870876.

Modha J., Kusel J.R. \& Kennedy M. A role for second messengers in the control of activation of the surface of Trichinella spiralis infective larvae. Molecular Biochemical Parasitology, 1995, 72, 141.

Narasimhan K., Basheer C., Bajic V.B. \& Swarup S. Enhancement of plant-microbe interactions using a rhizosphere metabolomics-driven approach and its application in the removal of polychlorinated biphenyls. Plant Physiology, 2003, $132,146$.

Olsen D.P., Phu D., Libby L.J.M., Cormier J.A., Montez K.M., RYDER E.F. \& Politz S.M. Chemosensory control of surface antigens switching in the nematode Caenorhabditis elegans. Genes, Brain and Behaviour, 2007, 6, 240-252.

PERRY R.N. An evaluation of types of attractants enabling plant-parasitic nematodes to locate plant roots. Russian Journal of Nematology, 2001, 13, 83-88.

Prior A., Jones J., Block V., Beauchamp J., McDermott L., CoOper A. \& Kennedy M. A surface-associated retinol- and fatty acid protein (Gp-FAR-1) from the potato cyst nematode Globodera pallida: lipid binding activities, structural analysis and expression pattern. Biochemistry Journal, 2001, 356, 387-394.

Proudfoot L., Kusel J.R., Smith H.V. \& Kennedy M. External stimuli and intracellular signalling in the modification of the nematode surface during transition to the mammalian host environment. Parasitology, 1993, 107, 559-566.

PROT J.C. Migration of plant-parasitic nematodes towards roots. Revue de Nématologie, 1980, 3, 305-318. 
Prusty R., Grisafi P. \& Fink G. The plant hormone indoleacetic acid induces invasive growth in Saccharomyces cerevisiae. Proceedings of the National Academy of Science of the USA, 2004,101, 4153-4157.

RoBinson F. Nematodes behaviour and migration through soil and host tissue, in: Basis of behaviour. Chen C., Chen S. \& Dickson D.W. (eds). Recent Nematology Topics, 2002, 331-401.

Robinson M.P., AtKinson H.J. \& PERry R.N. The influence of soil moisture and storage time on the motility, infectivity and lipid utilization of second stage juveniles of the potato cyst nematodes Globodera rostochiensis and G. Pallida. Revue de Nématologie, 1987, 10, 343-348.

Robertson L., Robertson W.M., Sobczak M., Helder J., Tetaud E., Ariyanayagam M.R., Fergusson M.A.J., Fairlamb A. \& Jones J. Cloning, expression and functional characterisation of a peroxiredoxin from the potato cyst nematode Globodera rostochiensis. Molecular and Biochemical Parasitology, 2000, 111, 41-49.

SAWHney R. \& Webster J.M. The role of plant growth hormones in determining the resistance of tomato plants to the root-knot nematodes, Meloidogyne incognita. Nematologica, 1975, 21, 95-103.

SCHultz J. Shared signals and the potential for phylogenetic espionage between plants and animals. Integrative and Comparative Biology, 2002, 42, 454-462.

Sharon E., Spiegel Y., Solomon R. \& Curtis R.H.C. Characterisation of Meloidogyne javanica surface coat using antibodies and their effect on nematode behaviour. Parasitology, 2002, 125, 177-185.

Spiegel Y. \& McClure M.A. The surface coat of plant-parasitic nematodes: chemical composition, origin and biological role. A review. Journal of Nematology, 1995, 27, 127134.

STAMPS W.T. \& LINT M. Interaction of intrinsic and extrinsic chemical cues in the behaviour of Bursaphelenchus xylophilus (Aphelenchida: Aphelenchoididae) in relation to its beetle vectors. Nematology, 2001, 3, 295-301.

Stewart G.R., Perry R.N. \& Wright D.J. Studies on the amphid specific glycoprotein gp-32 in different life-cycles stages of Meloidogyne species. Parasitology, 1993, 107, 573-578.

Timmermans B.C.G., Vos J., Stomph T.J., Van Nieuwburg J. \& VAN DER PUTTEN P.E.L. Field performance of Solanum sisymbriifolium, a trap crop for potato cyst nematodes. II. Root characteristics. Annals of Applied Biology, 2007, 150, 99106.

Trett M.W. \& Perry R.N. Functional and evolutionary implications of the anterior sensory anatomy of species of rootlesion nematode. Revue de Nématologie, 1985, 8, 341-355.

VON MENDE N. Invasion and migration behaviour of sedentary nematodes, in: Cellular and molecular aspects of plant-nematode interactions. Kluwer Academic Publishers, The Netherlands, 1997, 51-64.

Williamson V.M. \& Hussey R.S. Nematode pathogenesis and resistance in plants. Plant Cell, 1996, 8, 1735-1745.

Wuyts N., Swennen R. \& Waele D. Effects of plant phenylpropanoid pathway products and selected terpenoids and alkaloids on the behaviour of the plant parasitic nematodes
Radopholis similis, Pratylenchus penetrans and Meloidogyne incognita. Nematology, 2006, 8, 89-101.

Zasada I.A., Meyer S.L.F., Halbrendt J.M. \& Rice C. Activity of hydroxamic acids from Secale cereale against the plantparasitic nematodes Meloidogyne incognita and Xiphinema americanum. Phytopathology, 2005, 95, 1116-1121.

Zhao X., Schmitt M. \& Hawes M. Species-dependent effects of border cell and root tip exudates on nematode behaviour. Nematology, 2000, 90, 1239-1245.

ZuCKERMAN B.M. Hypotheses and possibilities of intervention in nematode chemoresponses. Journal of Nematology, 1983, 15, 173-182. 